ISSN 0103-5150

Fisioter. Mov., Curitiba, v. 25, n. 3, p. 481-488, jul./set. 2012 Licenciado sob uma Licença Creative Commons

\title{
Efeitos da aplicação do L.A.S.E.R. HeNe e do ultravioleta B no vitiligo
}

\author{
Application purposes of HeNe L.A.S.E.R. \\ and ultraviolet $B$ in vitiligo
}

\section{Ana Carolina Brandt de Macedo ${ }^{[a]}$, Camila Fátima de Oliveira ${ }^{[b]}$, Elaine Cristina Magalhães Silva ${ }^{[c]}$, Luciane Aparecida Straub de Andrade ${ }^{[d]}$}

[a] Fisioterapeuta, mestre em Engenharia Biomédica, doutoranda em Educação Física, docente do curso de Fisioterapia das Faculdades Integradas do Brasil (UniBrasil), Curitiba, PR - Brasil, e-mail: acbrandt@bol.com.br

[b] Aluna do curso de Fisioterapia das Faculdades Integradas do Brasil (UniBrasil), Curitiba, PR - Brasil, e-mail: camyfaty@yahoo.com.br

[c] Aluna do curso de Fisioterapia das Faculdades Integradas do Brasil (UniBrasil), Curitiba, PR - Brasil, e-mail: elaine.sjp@gmail.com

[d] Aluna do curso de Fisioterapia das Faculdades Integradas do Brasil (UniBrasil), Curitiba, PR - Brasil, e-mail: lu_straub@hotmail.com

\section{Resumo}

Introdução: $O$ vitiligo é uma doença crônica despigmentante da pele, ocasionada pela perda funcional dos melanócitos da epiderme. Este estudo teve como objetivo comparar os efeitos da aplicação do L.A.S.E.R. HeNe e do ultravioleta B em pacientes com vitiligo. Método: Foram selecionados 22 pacientes (11 mulheres e 11 homens), com idade entre 12 e 60 anos (31,68 16,29). Os pacientes foram distribuídos aleatoriamente, de acordo com a ordem de comparecimento no serviço e foram divididos randomicamente em dois grupos: o grupo A $(n=12)$, submetido à aplicação do L.A.S.E.R. HeNe, para o qual a técnica utilizada foi a pontual com aproximadamente $3 \mathrm{~cm}$ de distância entre cada ponto, perpendicularmente à pele, e o grupo $\mathrm{B}$ ( $n=10)$, exposto ao Ultravioleta B, aplicado de forma perpendicular com $10 \mathrm{~cm}$ de afastamento da pele do paciente. Para mensuração da área tratada, utilizou-se o programa AUTOCAD 2002, no qual foi delimitada a borda da mácula em fotos tiradas no início e no fim do tratamento. Resultados: Inicialmente, no grupo A, a área era de 4,87 $\mathrm{cm}^{2} \pm 3,70$ e, após a aplicação, foi de 4,15 $\mathrm{cm}^{2} \pm 2,89$, com diferença média de $0,72 \mathrm{~cm}^{2}$ $(1,89)$. No grupo B, verificou-se que antes da aplicação a média era de $5,36 \mathrm{~cm}^{2} \pm 6,36$ e, a após a aplicação, 
foi de 4,43 $\mathrm{cm}^{2} \pm 5,17$, com diferença média de $0,92 \mathrm{~cm}^{2} \pm 1,29$. Conclusão: Ambos os aparelhos obtiveram resultados significativos na redução da mácula, com maior redução no grupo da ultravioleta.

Palavras-chave: Vitiligo. Laser de gás. Terapia ultravioleta.

\section{Abstract}

Introduction: The Vitiligo is a chronic depigmentation illness of the skin caused by loss of functional melanocytes in the epidermis. This study's objective was to compare the effects of applying L.A.S.E.R. HeNe and ultraviolet $B$ in patients with vitiligo. Method: 22 patients (11 women and 11 men) aged 12-60 years $(31,68 \pm$ $16,29)$ were selected. Patients were randomly assigned, according to the order of attendance at the service and were divided randomly into two groups: group A $(n=12)$ subjected to the application of L.A.S.E.R. HeNe, where the technique used was punctual with about $3 \mathrm{~cm}$ distance between each point, perpendicular to the skin, and group $B(n=10)$ exposed to Ultraviolet B applied perpendicular $10 \mathrm{~cm}$ away from the patient's skin. For measurement of the treated area it was used the AUTOCAD program, which was bounded to the edge of the stain on photos taken at the beginning and at the end of treatment. Results: Initially, in group A the area was $4,87 \mathrm{~cm}^{2} \pm 3,70$, and after application it was $4,15 \mathrm{~cm}^{2} \pm 2,89$, with an average difference of $0,72 \mathrm{~cm}^{2} \pm$ 1 ,89. In group $B$ it was noted that before applying the average was $5,36 \mathrm{~cm}^{2} \pm 6,36$, and after application it was 4,43 $\mathrm{cm}^{2} \pm 5,17$, with average difference of $0,92 \mathrm{~cm}^{2} \pm 1,29$. Conclusion: Both devices achieved significant results in reducing the stain, with a greater reduction in the group of ultraviolet.

Keywords: Vitiligo. Gas laser. Ultraviolet therapy.

\section{Introdução}

0 vitiligo é uma doença crônica despigmentante que atinge a pele, com o surgimento de máculas brancas, ocasionadas pela perda funcional dos melanócitos da epiderme (1-5). Essa desordem pigmentar adquirida é comum em todas as raças, afetando pelo menos $1 \%$ do total da população antes dos 20 anos de idade $(4,6)$.

O curso clínico da doença é imprevisível, apresentando geralmente uma progressão lenta, com o aparecimento de novas lesões ou a expansão das lesões existentes. $\mathrm{O}$ vitiligo pode ser focal, segmentar, generalizado (vulgaris), ou mesmo universal $(2,7)$. A etiologia e patogenia exata da doença são desconhecidas. Existem teorias como as hipóteses neural, imune e autodestrutiva (6-10). A caracterização do vitiligo sugere que fatores predisponentes (genéticos) e precipitantes (ambientais) tornam uma pessoa mais suscetível à doença. Dentre os possíveis mecanismos que levam ao desaparecimento dos melanócitos funcionais, estão os imunomediados e os tóxicos como os mais prováveis (7). Evidências sugerem a ocorrência de um estresse oxidativo, levando à degeneração dos melanócitos $(3,11,12)$. A estimulação dos melanócitos da epiderme, tanto as células recém-chegadas da bainha externa da raiz quanto as residuais das células da pele, resulta na recuperação da patologia. Independentemente do mecanismo, (I) queratinócitos, (II) fibroblastos, melanoblastos (III) e melanócitos (IV) podem todos ser envolvidos na repigmentação do processo de vitiligo (5).

A repigmentação depende de melanócitos disponíveis a partir de três fontes possíveis: da unidade do folículo piloso, que é o principal fornecedor de células de pigmento; da borda das lesões de vitiligo; e dos melanócitos nas zonas afetadas despigmentadas (13).

0 tratamento do vitiligo ainda é um desafio terapêutico. Das modalidades disponíveis atualmente pode-se utilizar a fototerapia, que inclui o ultravioleta $B$ (UVB) e o L.A.S.E.R. HeNe $(2,12,14)$.

A radiação ultravioleta $B$ (RUV-B), na faixa de 290 a $320 \mathrm{~nm}$, é o comprimento de onda de maior efeito biológico e é absorvido por cromóforos endógenos. O UVB tem ação direta sobre os queratinócitos, induzindo alterações estruturais e funcionais por meio de fenômenos imunológicos e maior produção de citocinas imunossupressoras que estimulam a migração e proliferação dos melanócitos $(15,16)$. Indicações para a fototerapia com UVB são mais adequadas para psoríase, pitiríase rósea, foliculite pustulosa, pitiríase liquenóide crônica, controle do prurido e vitiligo $(15,17,18)$. 
O L.A.S.E.R. que pode ser utilizado para tratamento dessa patologia é o $\mathrm{HeNe}$, que é de baixa energia e emite radiação em espectro de luz visível (10). Estudos demonstram que, além de modulação dos queratinócitos, fibroblastos e melanoblastos, o tratamento induz a síntese do fator de melanócitos relacionados com o crescimento, estimulando a proliferação de melanócitos. A eficaz estimulação dos queratinócitos é provavelmente suficiente para induzir a repigmentação do vitiligo (5). Assim, este estudo teve como objetivo comparar os efeitos da aplicação do L.A.S.E.R. HeNe e do ultravioleta B em pacientes com vitiligo.

\section{Materiais e métodos}

Este trabalho foi realizado nas dependências da Clínica de Fisioterapia das Faculdades Integradas do Brasil (UNIBRASIL), em Curitiba. Recebeu aprovação do Comitê de Ética em Pesquisa da UNIBRASIL, Parecer n. 020/2009, de acordo com a Resolução CNS 196/96.

\section{Amostragem}

A pesquisa realizada tratou-se de um estudo longitudinal. Foram selecionados para o estudo 22 pacientes (11 mulheres e 11 homens), com idade entre 12 e 60 anos $(31,68 \pm 6,29)$. Os pacientes foram amplamente informados sobre a natureza do estudo e assinaram um termo de consentimento livre e esclarecido para participação, sendo que os menores de idade obtiveram autorização de seus responsáveis legais. Os pacientes foram distribuídos aleatoriamente, de acordo com a ordem de comparecimento no serviço, e foram divididos randomicamente em dois grupos: o grupo $A(n=12)$, submetido à aplicação do L.A.S.E.R., e o grupo B $(n=10)$, exposto ao Ultravioleta. Os atendimentos foram realizados duas vezes por semana, durante cinco semanas, totalizando dez atendimentos para cada paciente.

\section{Critérios de inclusão}

Como critérios de inclusão, foram selecionados os pacientes que apresentaram o diagnóstico da patologia de vitiligo.
Critérios de exclusão

Foram excluídos os voluntários que apresentaram patologias dermatológicas associadas, tempo de exposição solar maior que quatro horas por dia, uso de medicamentos e/ou que estivessem em outros tipos de tratamentos, como fototerapia, homeopatia e outros para o vitiligo que pudessem alterar os resultados do estudo.

\section{Avaliação clínica}

Durante a seleção, os pacientes foram submetidos à avaliação clínica, por meio de questionário desenvolvido para o estudo. 0 questionário foi composto por identificação do paciente e por perguntas como: 1 - Tempo de diagnóstico; 2 - Doenças associadas; 3 - Realização de outros tratamentos; 4 - Raça; 5 - Localização anatômica das máculas. Esse questionário foi desenvolvido para obter dados estatísticos, podendo ser correlacionado com o resultado do tratamento.

Procedimentos de intervenção

Para o tratamento do grupo A, foi utilizado o L.A.S.E.R. de baixa potência Hélio-Neônio ( $\mathrm{HeNe}$ ), com comprimento de onda de 632,8 nm (marca HTM Compact), densidade de energia de $6 \mathrm{~J} / \mathrm{cm}^{2}$, modo de emissão contínua durante 1 minuto e 15 segundos por ponto (esse tempo foi estipulado pelo equipamento). Para aplicação do L.A.S.E.R., foi escolhida uma mácula e feita a assepsia da pele com álcool $70 \%$, e a técnica utilizada foi a pontual, com aproximadamente $3 \mathrm{~cm}$ de distância entre cada ponto, perpendicularmente à pele. A escolha do L.A.S.E.R. HeNe foi em função de ter penetração mais superficial, atingindo a epiderme e ativando a produção dos melanócitos e de melanina (5).

Para o tratamento do grupo B, foi utilizado o Ultravioleta, com lâmpada fluorescente com radiação B (RUV-B) na faixa de 290 a $320 \mathrm{~nm}$. Primeiramente, os pacientes foram submetidos ao teste de Saidman, para verificar a dose do eritema mínimo, sendo que após 24 horas foi determinado o tempo de aplicação (15). Após a determinação do tempo, foi escolhida uma mácula e aplicado o UVB perpendicular com $10 \mathrm{~cm}$ de afastamento da pele 
do paciente. Foi escolhida a radiação ultravioleta B (RUV-B), na faixa de 290 a $320 \mathrm{~nm}$, por ter maior efeito biológico e estar associada à capacidade de produzir eritema, ativando a produção de melanócitos e repigmentando a pele (19).

\section{Registro das máculas}

Para registro dos resultados, foi utilizada uma régua para medir a extensão da mácula, como referência nas medidas realizadas no AUTOCAD da área a ser tratada, sendo depois fotografada com máquina da marca Olympus ${ }^{\circledR}$, numa distância de $10 \mathrm{~cm}$ da pele. As fotos foram tiradas antes e após o tratamento, para comparação dos resultados.

Para medição da área tratada, utilizou-se o programa AUTOCAD 2002, no qual foi delimitada a borda da mácula e, posteriormente, a escala utilizada (determinada pela régua posicionada ao lado do local); automaticamente, o programa calculou a área total. A Figura 1 mostra a delimitação da área realizada no AUTOCAD.

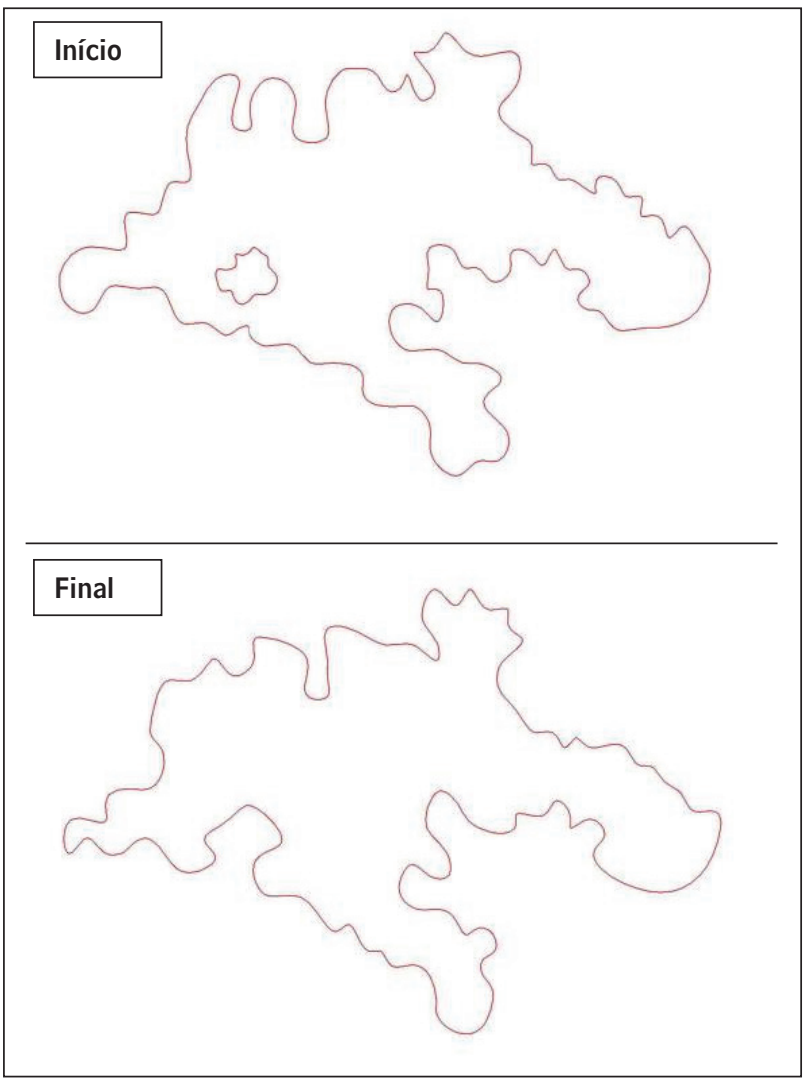

Figura 1 - Delimitação da borda da mácula no AUTOCAD Fonte: Dados da pesquisa.
A mensuração da área foi realizada nas fotos do início e do fim do tratamento.

Análise estatística

Os resultados das aplicações foram analisados estatisticamente utilizando-se o teste de Wilcoxon, com nível de significância $\mathrm{p}<0,05$.

\section{Resultados}

Doze pacientes participaram da pesquisa, sendo sete mulheres e cinco homens com idade média de $30,91 \pm 17,12$. Seis pacientes do grupo A e quatro do grupo $\mathrm{B}$ abandonaram o estudo, sendo seus dados desconsiderados para esta análise, permanecendo, assim, no grupo A ( $\mathrm{n}=6)$, idade de $12-46$ anos $(31,66$ $\pm 17,69)$ e, no grupo $B(n=6)$, idade de $13-60$ anos $(30,16 \pm 18,19)$.

Os resultados encontrados no questionário e aplicados para os grupos A e B estão ilustrados na Tabela 1. A média de idade de ambos os grupos foram similares e o sexo feminino foi predominante, porém foi maior no grupo A. A raça predominante do estudo foi a parda, sendo maior no grupo B. 0 tempo de diagnóstico $>10$ anos e o tratamento medicamentoso realizado anteriormente foram maiores em ambos os grupos, sendo que no grupo A houve tratamento homeopático e fototerapia. A maioria dos participantes não apresentou doenças associadas, e os locais mais acometidos pelo vitiligo foram MMSS, MMII e tronco, respectivamente.

O Gráfico 1 mostra o tamanho da mácula antes e após a aplicação do L.A.S.E.R. HeNe no grupo A. Inicialmente, a área média era de $4,87 \mathrm{~cm}^{2} \pm 3,70$ e, após o tratamento, era de $4,15 \mathrm{~cm}^{2} \pm 2,89$, com diferença de média $0,72 \mathrm{~cm}^{2} \pm 1,89$. A análise estatística evidenciou que houve diferença significativa entre as áreas médias das máculas do início e do fim da aplicação ( $p=0,007)$.0 Gráfico 2 demonstra os resultados encontrados no grupo B. Verifica-se que, antes da aplicação, a média era de $5,36 \mathrm{~cm}^{2} \pm$ 6,36 , e após ela, de $4,43 \mathrm{~cm}^{2} \pm 5,17$, com diferença média de $0,92 \mathrm{~cm}^{2} \pm 1,29$. A análise estatística também evidenciou que houve diferença significativa entre as áreas médias das máculas do início e do fim da aplicação $(p=0,02)$. 
Tabela 1 - Resultados do questionário aplicado para os grupos A e B

\begin{tabular}{|c|c|c|}
\hline \multicolumn{3}{|c|}{ Grupos } \\
\hline & $A$ & B \\
\hline \multicolumn{3}{|l|}{ IDADE (média) } \\
\hline & $31,66 \pm 17,69$ & $30,16 \pm 18,19$ \\
\hline \multicolumn{3}{|l|}{ Sexo } \\
\hline Feminino & 4 & 3 \\
\hline Masculino & 2 & 3 \\
\hline \multicolumn{3}{|l|}{ Raça } \\
\hline Brancos & 3 & 1 \\
\hline Pardos & 2 & 5 \\
\hline Negros & 1 & - \\
\hline \multicolumn{3}{|c|}{ Tempo de diagnóstico } \\
\hline 1 a 3 anos & 1 & 1 \\
\hline 4 a 5 anos & 2 & - \\
\hline 9 a 10 anos & - & 1 \\
\hline$>10$ anos & 3 & 3 \\
\hline \multicolumn{3}{|c|}{ Tratamentos realizados } \\
\hline Medicamentosos & 5 & 6 \\
\hline Homeopáticos & 2 & - \\
\hline Fototerapia & 1 & - \\
\hline \multicolumn{3}{|c|}{ Doenças associadas } \\
\hline Sim & 1 & 2 \\
\hline Não & 5 & 4 \\
\hline \multicolumn{3}{|c|}{ Localização das máculas } \\
\hline MMII & 4 & 5 \\
\hline MMSS & 5 & 5 \\
\hline Face & - & 4 \\
\hline Tronco & 2 & 3 \\
\hline
\end{tabular}

Fonte: Dados da pesquisa.

Os locais de aplicação nos grupos A e B estão ilustrados na Tabela 2. Pode-se observar que no grupo A (L.A.S.E.R.) a redução da mácula foi maior no antebraço e, no grupo B (UVB), foi maior na perna, com menor redução nas mãos. Também pode ser observado que em ambos os grupos houve um aumento da mácula na região do pé, após as dez aplicações.

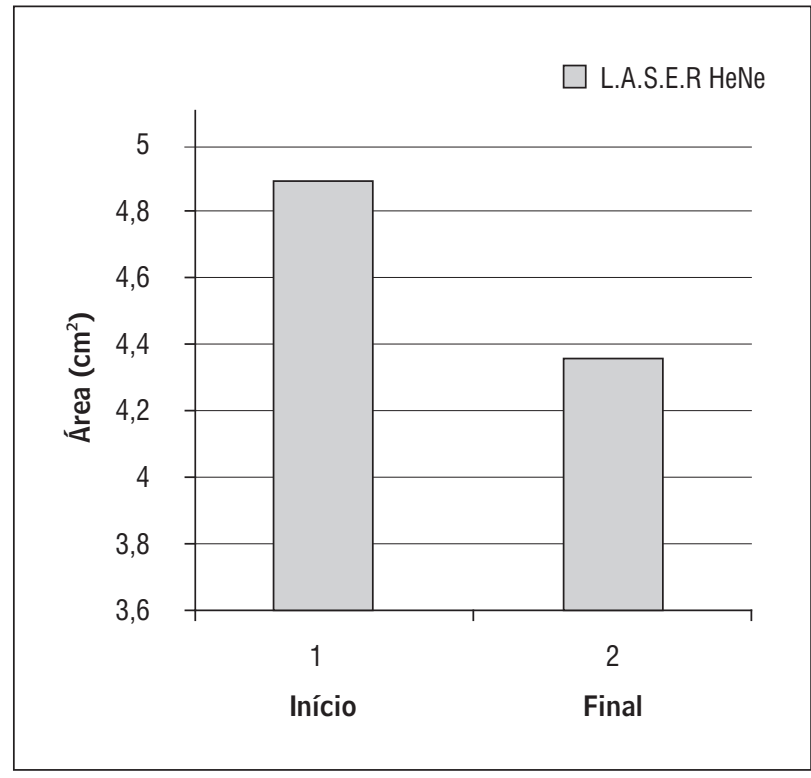

Gráfico 1 - Comparação do tamanho das máculas do grupo A no início e no fim do tratamento

Fonte: Dados da pesquisa.

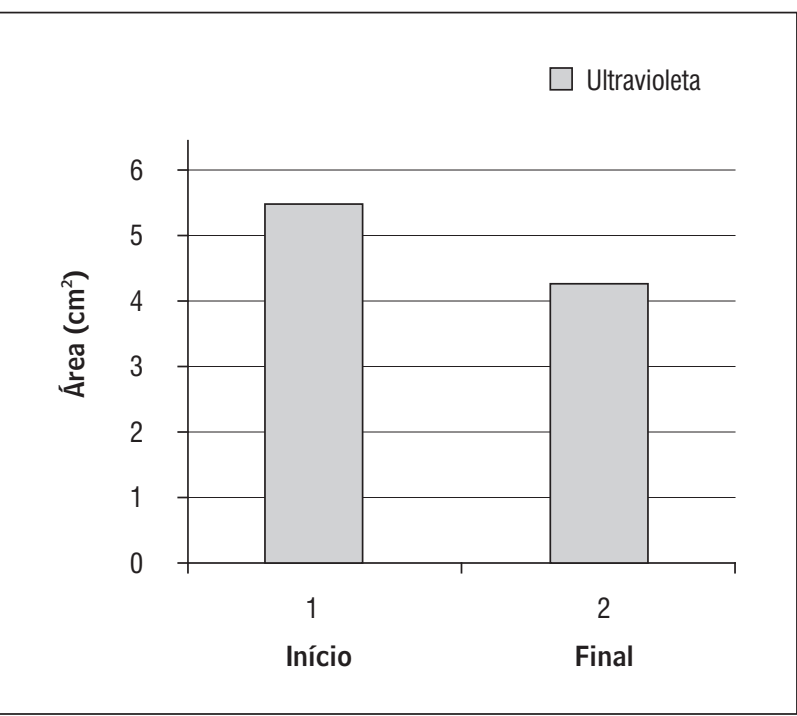

Gráfico 2 - Comparação do tamanho das máculas do grupo B no início e no fim do tratamento

Fonte: Dados da pesquisa.

\section{Discussão}

Nesse estudo, foi possível observar que houve uma diminuição da mácula do início para o fim do tratamento, com diferença média de $0,72 \mathrm{~cm}^{2} \pm 1,89$, após dez aplicações do L.A.S.E.R. HeNe, com análise estatística significante $(p<0,05)$. Yu et al. $(20)$, em seu estudo 
Tabela 2 - Local da aplicação do L.A.S.E.R. HeNe e do UVB nos grupos A e B

\begin{tabular}{lcccc}
\hline \multicolumn{5}{c}{ Local de aplicação } \\
\hline & A & $\begin{array}{c}\text { Média } \\
\text { (redução da mácula) }\end{array}$ & B & $\begin{array}{c}\text { Média } \\
\text { (redução da mácula) }\end{array}$ \\
\hline Mão & 1 & 0.364 & 3 & 0.313 \\
Antebraço & 2 & 2.218 & 1 & 1.751 \\
Tronco & 2 & 0.325 & - & - \\
Perna & - & - & 1 & 3.183 \\
Pé & 1 & -1.101 & 1 & -0.293 \\
\hline
\end{tabular}

Fonte: Dados da pesquisa.

in vitro com 30 pacientes portadores de vitiligo segmentar, com idade média de 22,7 anos, demonstraram que, após uma média de 16 sessões de tratamento com L.A.S.E.R. HeNe, houve um aumento na migração de melanócitos e repigmentação inicial notável. Concluíram que a irradiação do L.A.S.E.R. HeNe estimula a migração e a proliferação de melanócitos e a liberação mitógeno para o crescimento de melanócitos, podendo, também, recuperar as células danificadas, fornecendo um microambiente para induzir repigmentação no vitiligo.

A irradiação do L.A.S.E.R. mostra ter um efeito importante no desencadeamento da proliferação celular, diferenciação e apoptose em vários tipos celulares $(5,21)$. Em particular, o L.A.S.E.R. de baixa energia tem demonstrado um papel terapêutico em diversas situações, como na artrite reumatoide, na cicatrização de feridas, na nevralgia pós-herpética, na recuperação da lesão do nervo e no vitiligo $(20,21)$.

Tem sido relatado que o L.A.S.E.R. HeNe induz a proliferação de melanócitos e que esse tipo de tratamento tem efeitos estimulatórios (maior) e inibitórios (menor) nas células irradiadas (5).

Em relação ao tratamento com UVB, observou-se que também houve uma diminuição das máculas do início e do fim, com diferença média de $0,92 \mathrm{~cm}^{2}$ $\pm 1,29$, após dez aplicações, com análise estatística significante $(\mathrm{p}=0,02)$. No vitiligo, é provável que o mecanismo de repigmentação com UVB esteja relacionado à produção de mediadores inflamatórios na pele, que estimulam a migração e a proliferação dos melanócitos $(19,22,23,24)$.

Sitek et al. (25), após terem realizado tratamento com UVB por 12 meses, em 31 pacientes com vitiligo generalizado, mostraram que 11 pacientes tratados tiveram > 75\% de repigmentação, 19 pacientes apresentaram $>25 \%$ de repigmentação total e sete pacientes não obtiveram nenhum efeito durante todo os primeiros três meses, tendo interrompido o tratamento. Cinco pacientes que tiveram $>75 \%$ de repigmentação foram acompanhados e permaneceram estáveis após dois anos da cessação do tratamento.

Comparando os resultados dos dois grupos, o grupo A apresentou uma redução das máculas de $14,86 \%$, enquanto o grupo B obteve uma redução de $17,32 \%$ da área das máculas, demonstrando que o tratamento com UVB foi mais eficaz em relação ao L.A.S.E.R. HeNe nesses pacientes.

Neste estudo, não foram utilizados medicamentos e pomadas associadas ao tratamento, porém, estudos anteriores, realizados com UVB associado a pomada e/ou medicamentos e somente com o UVB demonstraram que o tratamento associado a outro método é mais eficaz, concluindo, portanto, que eles podem ser um método coadjuvante para o tratamento do vitiligo (17, $23,26,27)$. Middelkamp-Hup et al. (26) constataram que a repigmentação foi considerada clinicamente relevante em $72 \%$ dos casos de um grupo que fez combinação do UVB com leucotomos P. (cápsula antioxidante), em relação ao grupo que utilizou placebo e UVB. Já Fai et al. (11), em seu estudo com UVB, concluíram que a pomada não acrescenta nenhum benefício significativo sobre o UVB sozinho no tratamento do vitiligo.

Em relação aos locais de aplicação do grupo A, houve uma diminuição maior da mácula na região do antebraço, já no grupo B houve uma diminuição maior na região da perna e com menor redução nas mãos. Pode-se observar que em ambos os grupos houve um aumento maior na região do pé. 
Para que a repigmentação ocorra por estimulação, os melanócitos devem estar presentes em número adequado. É de especial importância enfatizar que os folículos pilosos estão ausentes nas palmas das mãos, nas plantas dos pés, nas mucosas ou semimucosas, e, portanto, essas áreas tornam-se particularmente refratárias a todas as terapias, pois o reservatório de melanócitos está faltando; no entanto, um padrão de repigmentação difuso é frequentemente observado $(13,20)$.

Elgoweini e Din (27) realizaram um estudo com UVB e concluíram que as melhores respostas foram obtidas no rosto, no tronco, nos braços e nas pernas. Por outro lado, mãos e pés mostraram repigmentação inferior a $25 \%$, em todos os casos. Isso pode acontecer por serem áreas pobres em folículos pilosos locais, onde estão presentes os melanócitos inativos, que constituem reserva melanocítica não afetada pelo vitiligo e são importantes no processo de repigmentação, o que ocorre mediante a estimulação de imunocitocinas e mediadores inflamatórios que agem como "sinais" para migração de melanócitos a partir dos folículos pilosos da pele normal (28-32).

\section{Conclusão}

Os resultados desta pesquisa, nas condições experimentais utilizadas e para a amostra estudada, permitiram concluir que os tratamentos com L.A.S.E.R. HeNe e UVB possibilitaram melhoras significativas, evidenciando a diminuição das máculas e que ambas as técnicas de tratamento obtiveram resultados satisfatórios. De acordo com os resultados obtidos, pode-se concluir que o UVB apresenta uma tendência a ser o tratamento de escolha para o vitiligo. Apesar de os pacientes tratados com o L.A.S.E.R. HeNe terem obtido uma diminuição significativa das máculas, o UVB apresentou melhores resultados em termos numéricos.

\section{Agradecimentos}

À orientadora, profa. Ana Carolina Brandt de Macedo, pelo apoio e pela dedicação à pesquisa. Aos técnicos de laboratório da Unibrasil, pela disponibilidade. A Kelly Cristina Magalhães, estatística que muito nos auxiliou. Aos pacientes portadores de vitiligo, pela confiança que depositaram na pesquisa e no tratamento. A todos que não foram citados nominalmente e que contribuíram para este trabalho.

\section{Referências}

1. Dell'Anna ML, Ottaviani M, Bellei B, Albanesi V, Cossarizza A, Rossi L. Membrane lipid defects are responsible for the generation of reactive oxygen species in peripheral blood mononuclear cells from vitiligo patients. J Cell Physiol. 2010;223(1):187-93. PMid:20049874.

2. Alghamdi KM. A survey of vitiligo management among dermatologists in Saudi Arabia. J Eur Acad Dermatol Venereol. 2009;23:1282-8. doi:10.1111/j.1468-3083.2009.03310.x.

3. Anbar TS, Westerhof W, Abdel-Rahman AT, Ewis AA, ElKhayyat MA. Effect of one session of ER: YAG LASER ablation plus topical 5 Fluorouracil on the outcome of short-term NB-UVB phototherapy in the treatment of non-segmental vitiligo: a left-right comparative study. Photodermatol Photoimmunol Photomed. 2008;24(6):322-9. doi:10.1111/ j.1600-0781.2008.00385.x.

4. Choi S, Kim DY, Whang SH, Lee JH, Hann SK, Shin YJ. Quality of life and psychological adaptation of Korean adolescents with vitiligo. Eur Acad Dermatol Venereol. 2010;24(5):5249. doi:10.1111/j.1468-3083.2009.03452.x.

5. Lan CCE, Wu CS, Chiou MH, Chiang TY, Yu HS. Low-energy helium-neon laser induces melanocyte proliferation via interaction with type IV collagen: visible light as a therapeutic option for vitiligo. Br J Dermatol. 2009;161(2):273-80. doi:10.1111/j.1365-2133.2009.09152.x.

6. Farajzadeh SSMD, Daraei ZMD, Esfandiarpour IMD. The efficacy of pimecrolimus $1 \%$ cream combined with microdermabrasion in the treatment of nonsegmental childhood vitiligo: a randomized placebo-controlled study. Pediatr Dermatol. 2009;26(3):286-91. doi:10.1111/j.15251470.2009.00926.x.

7. Lo YH, Cheng GS, Huang CC, Chang WY, Wu CS. Efficacy and safety of topical tacrolimus for the treatment of face and neck vitiligo. J Dermatol. 2010;37(2):125-9. doi:10.1111/ j.1346-8138.2009.00774.x.

8. Petsoglou SB, Wittal JLR. A randomized, double-blinded, placebo-controlled trial of pseudocatalase cream and narrowband ultraviolet $\mathrm{B}$ in the treatment of vitiligo. Br J Dermatol. 2009;161(4):910-7. doi:10.1111/j.13652133.2009.09252.x.

9. Khan R, Satyam A, Gupta S, Sharma VK, Sharma A. Circulatory levels of antioxidants and lipid peroxidation in indian patients with generalized and localized vitiligo. Arch Dermatol Res. 2009;301(10):731-7. doi:10.1007/s00403-009-0964-4.

10. Ferreira MC, Gameiro J, Nagib PR, Brito VN, Vasconcellos Eda C, Verinaud L. Effect of low intensity helium-Neon (HeNe) Laser irradiation on experimental paracoccidioidomycotic wound healing dynamics. Photochem Photobiol. 2009;85(1):227-33. doi:10.1111/j.17511097.2008.00423.x. 
11. Fai D, Cassano N, Vena GA. Narrow-band UVB phototherapy combined with tacrolimus ointment in vitiligo: a review of 110 patients. Eur Acad Dermatol Venereol. 2007;916-20.

12. Taher ZA, Lauzon G, Maguiness S, Dytoc MT. Analysis of interleukin-10 levels in lesions of vitiligo following treatment with topical tacrolimus. Br J Dermatol. 2006;161(3):654-9. doi:10.1111/j.1365-2133.2009.09217.x.

13. Falabella R. Vitiligo and the melanocyte reservoir. Indian J Dermatol. 2009;54(4):313-8. doi:10.4103/00195154.57604.

14. Fongers A, Wolkerstorfer A, Nieuweboer-Krobotova L, Krawczyk P, Tóth GG, van Der Veen JP. Long-term results of 2-mm punch grafting in patients with vitiligo vulgaris and segmental vitiligo: effect of disease activity. Br J Dermatol. 2009;161(5):1105-11. doi:10.1111/j.13652133.2009.09367.x.

15. Agne JE. Eletroterapia: teoria e prática. Santa Maria: Orium; 2005.

16. Yang YS, Cho HR, Ryou JH, Lee MH. Clinical study of repigmentation patterns with either narrow-band ultraviolet B (NBUVB) or $308 \mathrm{~nm}$ excimer laser treatment in Korean vitiligo patients. Int J Dermatol. 2010;49(3):317-23. doi:10.1111/j.1365-4632.2009.04332.x.

17. El Mofty M, Mostafa W, Esmat S, Youssef R, Azzam O, Hunter N. Narrow band ultraviolet B $311 \mathrm{~nm}$ in the treatment of vitiligo: two right-left comparison studies. Photodermatol Photoimmunol Photomed. 2006;22(1):6-11. doi:10.1111/j.1600-0781.2006.00189.x.

18. Yashar SS, Gielczyk R, Scherschun L. Narrow-band ultraviolet $\mathrm{B}$ treatment for vitiligo, pruritus, and inflammatory Dermatoses. Photodermatol Photoimmunol Photomed. 2003;19:164-8. doi:10.1034/j.1600-0781.2003.00039.x.

19. Casacci M, Thomas P, Pacifico A, Bonnevalle A, Paro Vidolin A, Leone G. Comparison between 308-nm monochromatic excimer light and narrowband UVB phototherapy (311-313 $\mathrm{nm}$ ) in the treatment of vitiligo - a multicentre controlled study. J Eur Acad Dermatol Venereol. 2007;21(7):956-63. doi:10.1111/j.1468-3083.2007.02151.x.

20. Yu HS, Wu CS, Yu CL, Kao YH, Chiou MH. Helium neon laser irradiation stimulates migration and proliferation in melanocytes and induces repigmentation in segmental-typevitiligo. J Invest Dermatol. 2003;120(1):56-64. doi:10.1046/j.1523-1747.2003.12011.x.

21. Lan CC, Wu CS, Chiou MH, Hsieh PC, Yu HS. Low-energy helium-neon laser induces locomotion of the immature melanoblasts and promotes melanogenesis of the more differentiated melanoblasts: recapitulation of vitiligo repigmentation in vitro. J Invest Dermatol. 2006;126(9):2119-26. doi:10.1038/sj.jid.5700372.
22. Cestari TF, Pessato S, Correa GP. Phototherapy - clinical indications. An Bras Dermatol. 2007;82(1):5-6.

23. Dell'Anna ML, Mastrofrancesco A, Sala R, Venturini M, Ottaviani M, Vidolin AP, et al. Antioxidants and narrow bandUVB in the treatment of vitiligo: a double-blind placebo controlled trial. Clin Exp Dermatol. 2007;32(6):631-6. doi:10.1111/j.1365-2230.2007.02514.x.

24. Parsad D, Kanwar AJ, Kumar B. Psoralen-ultraviolet A vs. narrow-band ultraviolet B phototherapy for the treatment of vitiligo. Eur Acad Dermatol Venereol. 2006;20(2):175-7. doi:10.1111/j.1468-3083.2006.01413.x.

25. Sitek JC, Loeb M, Ronnevig JR. Narrowband. UVB therapy for vitiligo: does the repigmentation last? Eur Acad Dermatol Venereol. 2007;21(7):891-6. doi:10.1111/j.14683083.2007.01980.x.

26. Middelkamp-Hup MA, Bos JD, Rius-Diaz F, Gonzalez S, Westerhof W. Treatment of vitiligo vulgaris with narrowband UVB and oral Polypodium leucotomos extract: a randomized double-blind placebo-controlled study. Eur Acad Dermatol Venereol. 2007;21(7):942-50. doi:10.1111/ j.1468-3083.2006.02132.x.

27. Elgoweini MM, Din NN. Response of vitiligo to narrowband ultraviolet B and oral antioxidants. J Clin Pharmacol. 2009;49:852-5. doi:10.1177/0091270009335769.

28. Barros JA, Filho MSAC, Martins LC. Vitiligo: histological and clinical evaluation after sequential curettage. An Bras Dermatol. 2007;82(4):327-35. doi:10.1590/S036505962007000400005 .

29. Pianigiani E, Risulo M, Andreassi A, Taddeucci P, Ierardi F, Andreassi L. Autologous epidermal cultures and narrow-band ultraviolet $\mathrm{b}$ in the surgical treatment of vitiligo. Dermatol Surg. 2005;31(2):155-9. doi:10.1111 /j.1524-4725.2005.31036.

30. Brazzelli V, Antoninetti M, Palazzini S, Barbagallo T, de Silvestri A, Borroni G. Critical evaluation of the variants influencing the clinical response of vitiligo: study of 60 cases treated with ultraviolet B narrowband phototherapy. Eur Acad Dermatol Venereol. 2007;21:1369-74. doi:10.1111/ j.1468-3083.2007.02278.x.

31. Steiner D. Vitiligo. An Bras Dermatol. 2004;79(3):335-51.

32. Welsh 0, Herz-Ruelas ME, Gómez M. Therapeutic evaluation of UVB-targeted phototherapy in vitiligo that affects less than $10 \%$ of the body surface area. Int J Dermatol. 2009;48:529-34. doi:10.1111/j.1365-4632.2009.03928.x.

Recebido: 10/10/2010

Received: 10/10/2010

Aprovado: $18 / 05 / 2011$ Approved: 05/18/2011 